\title{
The Effective Enhancement of Power System Security by Flexible Transmission Line Impedance with Minimal Rescheduling of Generators
}

\author{
Karuppasamy Muthulakshmi ${ }^{1}$, Rajamanickam Manickaraj Sasiraja², \\ Selvarajan Mukesh Muthu ${ }^{2}$ \\ ${ }^{1}$ Department of Electrical and Electronics Engineering, Kamaraj College of Engineering and Technology, \\ Virudhunagar, Tamilnadu, India \\ ${ }^{2}$ Department of Electrical and Electronics Engineering, Anna University Regional Campus Madurai, Madurai, \\ Tamilnadu, India \\ Email: muthusashi@gmail.com,rmseee@autmdu.ac.in,mukeshmuthu13@gmail.com
}

Received 15 March 2016; accepted 25 April 2016; published 28 April 2016

Copyright (C) 2016 by authors and Scientific Research Publishing Inc.

This work is licensed under the Creative Commons Attribution International License (CC BY). http://creativecommons.org/licenses/by/4.0/

(c) (i) Open Access

\begin{abstract}
After the digital revolution, the power system security becomes an important issue and it urges the power producers to maintain a well secured system in order to supply a quality power to the end users. This paper presents an integrated Corrective Security Constrained Optimal Power Flow (CSCOPF) with Flexible Transmission Line Impedance (FTLI) to enhance the power system security. The corrective approach of SCOPF is chosen, because it allows the corrective equipment to bring back the system to a stable operating point and hence, it offers high flexibility and better economics. The concept of FTLI arises from the ability of FACTS devices such as Thyristor Controlled Series Capacitor (TCSC), which can vary the line reactance to a certain extent. An enhanced security can be achieved by incorporating FTLI into the CSCOPF problem, since the power flow in a system is highly dependent on the line reactance. FTLI based CSCOPF can reduce the amount of rescheduling of generators, but it will result in an increased number of variables and thus, the complexity to the optimization process is increased. This highly complex problem is solved by using nonlinear programming. The AC based OPF model is preferred, since the corrective security actions require highly accurate solutions. IEEE 30 bus system is used to test the proposed scheme and the results are compared with the traditional CSCOPF. It can be seen that the proposed idea provides a notable improvement in the reduction of cost incurred for restoring the system security.
\end{abstract}

\section{Keywords}

Flexible Transmission Line Impedance, Power System Security, Security Constrained Optimal

How to cite this paper: Muthulakshmi, K., Sasiraja, R.M. and Mukesh Muthu, S. (2016) The Effective Enhancement of Power System Security by Flexible Transmission Line Impedance with Minimal Rescheduling of Generators. Circuits and Systems, 7, 381-389. http://dx.doi.org/10.4236/cs.2016.74033 


\section{Power Flow, Rescheduling of Generators}

\section{Introduction}

In the contemporary power scenario, the power system security has obtained much attraction due to the rapid increase in the power demand. Due to the increase in the consumption of electrical energy over the years, the generation has to be increased accordingly, in order to obtain a balanced system. But, the existing transmission system is designed to carry a certain amount of power only. However, the violation in the prescribed limit will result in a damage of transmission equipment and imposes a threat to overall security of the system. Either new line(s) should be added to the system or the existing transmission system must be used more efficiently to meet the increase in load demand and to preserve the security. The addition of new line is often impractical, because it is a very time-consuming task and sometimes becomes impossible with environmental limitations. Therefore, the efficient use of existing lines provides a better solution and hence, this work involves such an approach to enhance the system security. The problem of security is often incorporated with optimal power flow to achieve an added economical advantage and termed as Security Constrained Optimal Power Flow (SCOPF). There are two major types of SCOPF namely, Preventive SCOPF (PSCOPF) and Corrective SCOPF (CSCOPF). PSCOPF tries to obtain a normal state power flow solution, which is also feasible for all contingency conditions being considered with minimum cost [1]. It leaves the option to adjust controllable equipment, which makes the solution inflexible and may result in a high operating cost. This approach fails, when a feasible solution, satisfying both normal and contingency constraints, is absent. Hence, PSCOPF is not an attractive solution to the problem of power system security.

CSCOPF allows varying the control variables by adjusting the control equipment after a contingency occurrence [2]. In comparison, CSCOPF is more flexible and thus, may achieve a lower operating cost. However, the CSCOPF model also has its own difficulties. It can be seen that CSCOPF model may involve in the rescheduling of a large amount of real power, which increases the operational complexity and the cycling costs of the generators. Apart from these disadvantages, the operational economics and flexible quality of CSCOPF make it as a better approach. The above considerations motivate in deriving a new corrective SCOPF model to achieve the goal of minimizing the rescheduling of generators. Certain literatures [3]-[5] introduce the use of devices that are capable of providing the corrective control for preserving security, such as phase shifters [3] and power routers [4]. But, it is seen that phase shifter adjustment under given contingency may fail to converge. Several techniques are proposed as modified formulations and the new solution methods are helpful to solve the CSCOPF problem more effectively. Such a new formulation is towards the minimal corrections of control variables [6] and a hybrid method, which combines the evolutionary algorithm and interior point technique and also provides deterministic solutions [7].

However, the procedures that are discussed so far have considered the transmission elements as fixed resources. But, by installing more flexible elements such as, FACTS devices, the operation becomes more flexible and the transmission line impedances can be changed by most of the FACTS devices. But, the variation in the characteristics of transmission lines may affect the operation of protective devices [8]. A new hybrid GA approach has been proposed to solve DC-OPF, which is incorporated with FACTS devices [9]. Several literatures demonstrate the advantages that are provided by considering the flexibility offered by the FACTS devices. Notably, a method is presented to utilize variable impedance based FACTS devices for power system optimization [10]. An OPF model with consideration of Flexible Transmission Line Impedance (FTLI) is provided by Ding et al. [11].

\section{Proposed Work}

These works validate the cost reduction alone, when incorporating flexible Line impedance in OPF, but don't consider this flexibility as a viable solution to other power system problems such as, security, congestion management etc. Hence, this work proposes the use of FTLI to enhance the security of power system by a corrective approach. First, the problem is formulated into an AC-OPF for real time feasibility then, the impedance of transmission lines are taken as a variable and incorporated into that model. The nonlinear programming tech- 
nique is used to solve this proposed problem by using MATPOWER [12] environment in MATLAB.

The remaining structure of this manuscript is given as follows. Section 2 describes about FTLI. Section 3 presents the formulation of the CSCOPF. Section 4 provides the solution methodology to solve the CSCOPF problem. Section 5 presents the results obtained from the traditional and proposed CSCOPF method and comparisons, discussions related to it. Section 6 concludes the overall work.

\section{Flexible Transmission Line Impedance (FTLI)}

Traditionally, the transmission system is treated as a static component of a power system, except during the maintenance or outage conditions. However, the operation of power system can be made more flexible by installing more flexible elements, such as FACTS. It is known that the transmission line impedances can be changed by FACTS devices, such as TCSC to increase the transfer capacity. In near future, it is expected that sophisticated components can be developed and employed in power systems with advancements by further research in the area of materials and sensors, adaptable and economically viable devices such as piezo-resistor and thermistor impedance. These advanced components will allow the system operator to vary the transmission line impedance smoothly and make the power system with flexible impedance transmission lines. In this paper an optimal power flow model with the consideration of such flexible transmission line impedances (FTLIs) is proposed to achieve a better operational economics and enhanced security. The incorporation of FTLI means the inclusion of all line reactances as control variables in the optimization process. So, in the case of the IEEE 30 bus system, which has 41 branches it involves the addition of another 41 control variables and they are varied optimally along with other control variables (i.e., real power output of generators). The mathematical model of the proposed FTLI based CSCOPF is discussed in the subsequent section. This work is based on a futuristic view of the transmission system that can dynamically change its nature with respect to the changes in system state, rather than being static. This could impart commendable controllability and thus, provides a higher security to the system.

\section{Formulation of Traditional and Proposed CSCOPF}

Here, the traditional C-SCOPF refers as CSCOPF without considering FTLI (i.e. without having line reactance as a variable). Traditional CSCOPF involves in the adjustment of control variables, such as real power output of generator, transformer tap settings, etc. The major control action that is widely followed is the rescheduling of generator output. The mathematical formulation of a CSCOPF without FTLI is done and they are presented below. The objective function is the minimization of a function and it is given in Equation (1). The various constraints that are involved in this problem formulation are presented in Equations (2)-(7).

$$
\begin{aligned}
& \text { Objective function: Minimize } f(z) \\
& \text { Equality constraints: } g(z)=0
\end{aligned}
$$

Inequality constraints:

$$
h(z) \leq 0
$$

where,

$$
Z=\left[\begin{array}{c}
\theta \\
V_{m} \\
P_{g} \\
Q_{g}
\end{array}\right]
$$

where,

$\theta:$ Vector of all bus angles except slack bus;

$V_{m}$ : Vector of all bus voltage magnitudes;

$P_{g}$ : Vector of real power outputs of all generators;

$Q_{g}$ : Vector of reactive power outputs of all generators.

Depending upon the application, the objective function can be taken as cost or loss function. In this work, the minimization of the cost incurred in preserving security is taken as the objective. The equality constraints con- 
stitute the real and reactive power balance equations of the system as given in Equation (5).

where,

$$
g(z)=\left\{\begin{array}{lc}
P_{k}(z)-P_{k}^{\text {net }}=0 & \forall k \in p v, p q \\
Q_{k}(z)-Q_{k}^{\text {net }}=0 & \forall k \in p v
\end{array}\right.
$$

$P_{k}^{\text {net }}$ : Specified real power injection at bus $k$;

$Q_{k}^{\text {net }}$ : Specified reactive power injection at bus $k$;

$P_{k}(z)$ : Calculated real power injection at bus $k$;

$Q_{k}(z)$ : Calculated reactive power injection at bus $k$.

Whereas, the inequality constraints constitute the MVA flow limits (security constraints) on the branches, upper and lower bounds on the control variables and it is given in Equation (6).

$$
h(z)= \begin{cases}S_{f l o w, i}(z) \leq S_{\max , i} & \forall i=1,2, \cdots, N_{b r} \\ z_{\min } \leq z \leq z_{\max } & \forall z\end{cases}
$$

where,

$S_{\text {flow }, i}(z)$ : Apparent power flow in line $i$;

$S_{\text {max }, i}$ : Maximum allowed apparent power flow in line $i$;

$z_{\min }$ : Vector of lower bound values of variable vector $z$;

$z_{\max }$ : Vector of higher bound values of variable vector $z$.

The proposed CSCOPF involves adjustment of control variables, such as real power output of generator, transformer tap settings, along with the line reactance. The adjustment of line reactance just after an outage, which minimizes the rescheduling of generators, is the major control action followed here. The formulation of the proposed CSCOPF is similar to the formulation of the traditional CSCOPF as discussed above, except considering the line reactance as a variable and it is shown below.

where,

$$
Z=\left[\begin{array}{c}
\theta \\
V_{m} \\
P_{g} \\
Q_{g} \\
x_{L}
\end{array}\right]
$$

$x_{L}$ : Vector of all line reactance.

All the Equations (1)-(3) and Equations (5)-(6) are applied to the proposed CSCOPF formulation, but the new $z$ is given by the Equation (7).

\section{Solution Methodology}

Today, most of the market based dispatch procedures are executed using a DC model. Therefore, DC model based variation of line reactance (FACTS settings) seems to be compatible with the current practices. However, AC-based modeling of FACTS adjustment is essential for the real-time contingency analysis, where FTLI can be used to perform corrective actions. So, the AC model is chosen for this proposed CSCOPF problem and is solved by using interior point method. First, the state and cost of the system are derived for the operation of the system under normal state. The performance index for each line is calculated by using the formula as given in Equation (8). Then, an outage is assumed on the selected line and the new state of the system and its cost are derived after optimal rescheduling. The deviation in cost due to rescheduling is also recorded for the comparison with the results of proposed method. Normally, a contingency analysis is carried out on the system in order to perform a SCOPF. The performance indices (PI) of all lines are obtained by assuming the outage of various lines and then, performing a power flow. The lines with higher performance index, whose outage would cause severe impacts on the system, are considered as various cases in this analysis. The formula for the calculation of performance index of $j$ th line is given by,

$$
\operatorname{PI}(j)=\sum_{i=1}^{N_{b=1}}\left(\frac{S_{f(0 w, i j}}{S_{\max , i}}\right)^{2}
$$


where,

$S_{\text {flow }, i j}$ : Apparent power flow on line $i$ after an outage on line $j$;

$N_{b r}$ : Number of lines.

The interior point method is a gradient based iterative procedure and that is used to solve linear and nonlinear convex optimization problems. The CSCOPF problem consists of quadratic objective function (i.e. the cost function), nonlinear equality and inequality constraints. Therefore, it lies in the class of nonlinear programming (NLP) problem and thus, the interior point method is used. In this work, the formulated problem is solved by using the "fmincon" function, which is used to solve linear and nonlinear optimization problems in MATLAB.

\section{Results and Discussion}

The simulation tests are performed in IEEE 30 bus system [12] to verify the success of this proposed method. The single line diagram of test system is shown in Figure 1, which consists of 6 generators, 24 load buses and 41 branches. The essential codes are developed in MATLAB release 2014 with Intel i5 processor equipped with 6 GB RAM.

The PI of all the 41 lines are calculated as described above with the help of power flow and it is as given in Table 1. Once the PI of all lines is calculated then, the branches are sorted in the decreasing order of performance index. Hence, the outage of line $1-2$ is chosen for the further analysis.

The top $\mathrm{n}$ branches in the sorted list are chosen for further analysis. A plot of performance index versus branch number is shown in Figure 2. From the list of performance indices the top three branches are chosen for performing the contingencies. The system was initially scheduled without considering outage and then, an outage of the selected line was assumed. Then, the system was rescheduled by means of corrective action. The line, which is connected between the buses 1 and 2, has higher value of PI and hence, the outage of line 1 - 2 is considered for the analysis. In order to verify the effectiveness of this proposed approach two cases are considered as follows.

CASE 1A: In this case, the outage line is line number 1 , which connects the buses 1 and 2 . The generation and the associated cost corresponding to before and after an outage are given in Table 2. The total generation cost before the outage and after the outage are $8915.04 \$ / h r$ and $9490.38 \$ / h r$, respectively. The cost deviation between before and after the outage is $575.34 \$ / \mathrm{hr}$. It is expected that there is a notable reduction in generation cost after employing FTLI by considering the line reactance as a control variable.

It can be noted that rescheduling introduces an increase in the operating cost of $575.34 \$ / \mathrm{hr}$. The system is then solved by considering FTLI i.e. the line reactance is considered as a variable. The results before and after outage for this consideration are tabulated. As the line reactance is being taken as a variable, it is bounded in a

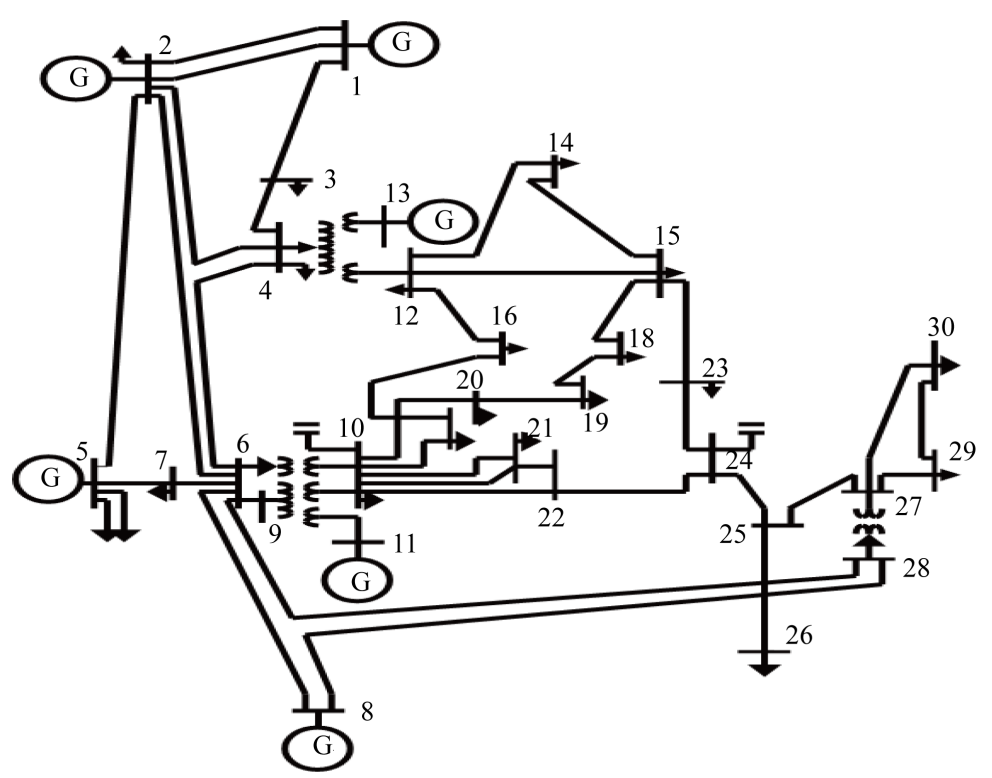

Figure 1. Single line diagram of IEEE-30 bus system. 
Table 1. Contingency ranking based on PI.

\begin{tabular}{|c|c|c|c|c|c|}
\hline Sl. No. & Outage of line & PI & Sl. No. & Outage of line & PI \\
\hline 1 & $1-2$ & 22.5578 & 22 & $12-14$ & 10.4877 \\
\hline 2 & $2-5$ & 17.5271 & 23 & $10-17$ & 10.4576 \\
\hline 3 & $4-12$ & 17.3503 & 24 & $25-27$ & 10.3775 \\
\hline 4 & $28-27$ & 15.4378 & 25 & $10-22$ & 10.3688 \\
\hline 5 & $1-3$ & 14.1156 & 26 & $22-24$ & 10.3627 \\
\hline 6 & $3-4$ & 13.9107 & 27 & $15-18$ & 10.2438 \\
\hline 7 & $4-6$ & 12.9821 & 28 & $9-11$ & 10.2425 \\
\hline 8 & $6-8$ & 12.1546 & 29 & $16-17$ & 10.1778 \\
\hline 9 & $9-10$ & 11.9785 & 30 & $29-30$ & 10.1759 \\
\hline 10 & $6-9$ & 11.9019 & 31 & $12-13$ & 10.1719 \\
\hline 11 & $10-20$ & 11.7978 & 32 & $2-4$ & 10.1667 \\
\hline 12 & $2-6$ & 11.5760 & 33 & $23-24$ & 10.1439 \\
\hline 13 & $6-28$ & 11.3785 & 34 & $8-28$ & 10.1304 \\
\hline 14 & $12-15$ & 11.2342 & 35 & $21-22$ & 10.1153 \\
\hline 15 & $19-20$ & 11.1419 & 36 & $14-15$ & 10.0778 \\
\hline 16 & $27-30$ & 11.0040 & 37 & $18-19$ & 10.0185 \\
\hline 17 & $10-21$ & 10.8074 & 38 & $24-25$ & 10.0050 \\
\hline 18 & $15-23$ & 10.5931 & 39 & $5-7$ & 9.7258 \\
\hline 19 & $6-10$ & 10.5742 & 40 & $6-7$ & 9.5125 \\
\hline 20 & $12-16$ & 10.5440 & 41 & $25-26$ & 9.4631 \\
\hline 21 & $27-29$ & 10.5016 & - & - & - \\
\hline
\end{tabular}

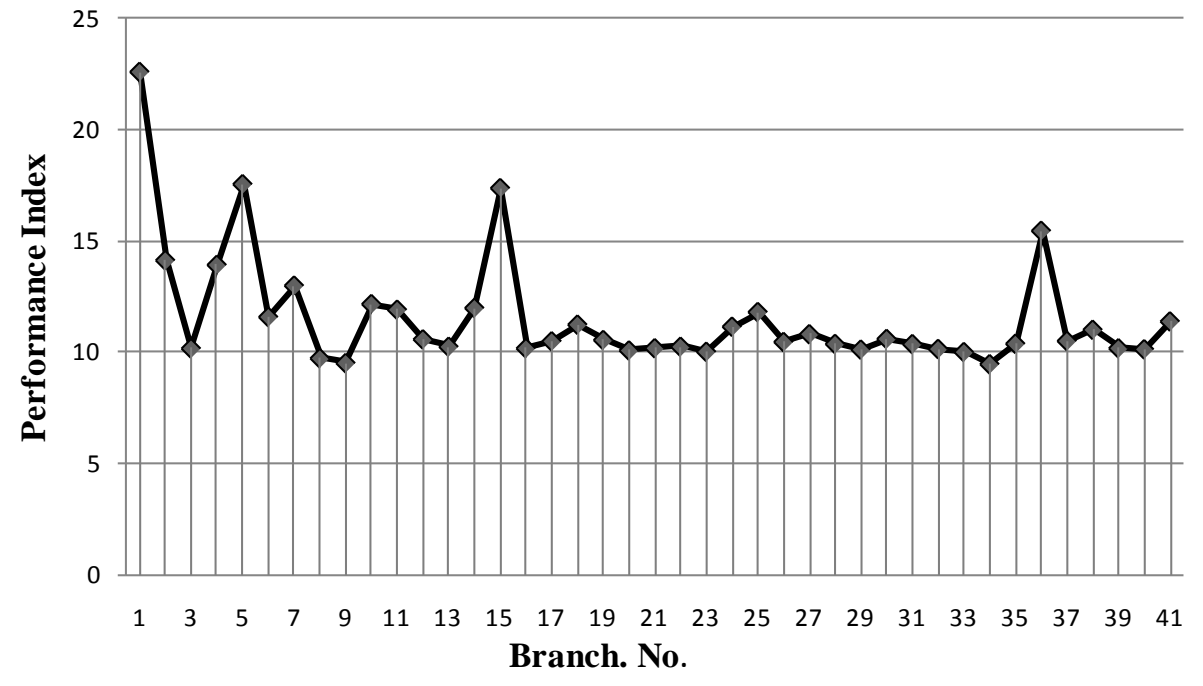

Figure 2. Performance index vs. branch number. 
Table 2. Generation and cost output for case 1A.

\begin{tabular}{|c|c|c|c|c|c|}
\hline \multirow{2}{*}{ Gen. Bus No. } & \multicolumn{2}{|c|}{ Before the outage } & \multicolumn{2}{|c|}{ After the outage } & \multirow{2}{*}{$\begin{array}{l}\text { Cost deviation } \\
\qquad(\$ / h r)\end{array}$} \\
\hline & Generation (MW) & Cost $(\$ / h r)$ & Generation (MW) & Cost $(\$ / h r)$ & \\
\hline 1 & 199.28 & \multirow{6}{*}{8915.04} & 129.88 & \multirow{6}{*}{9490.38} & \multirow{6}{*}{575.34} \\
\hline 2 & 37.22 & & 40.75 & & \\
\hline 5 & 35.99 & & 66.21 & & \\
\hline 8 & 15.09 & & 29.69 & & \\
\hline 11 & 6.33 & & 24.03 & & \\
\hline 13 & 0 & & 3.98 & & \\
\hline
\end{tabular}

certain range given by $[1-\alpha, 1+\alpha]$, where $\alpha$ is taken as $20 \%$.

CASE 1B: In this case, the line 1, which connects the buses 1 and 2, is considered as out of service and the system is rescheduled along with FTLI (i.e. considering line reactance as a variable). The results are tabulated in Table 3 and the cost deviation is 533.20 \$/hr.

It can be seen from Table 3 that the total generation cost for all the generators is $8915.04 \$ / \mathrm{hr}$ and the same after the outage is $9448.23 \$ / \mathrm{hr}$. The cost deviation in this case is identified as $533.20 \$ / \mathrm{hr}$. But, the cost deviation in the earlier case i.e. without considering the FTLI is $575.34 \$ / \mathrm{hr}$. But, the cost deviation after considering the line reactance as control variable gets reduced. The reason for this phenomenal reduction in cost deviation is the total generation cost reduction, when FTLI is employed after the outage. Because, the total generation costs after the outage without and with FTLI are 9490.38 \$/hr and $9448.23 \$ / \mathrm{hr}$, respectively.

The control action against this line outage is made easy by considering the line reactance as a flexible control variable. Hence, the action of real power allocation to all the participating generators is easily accomplished with the reduced level of total generation cost. This reduction in cost deviation shows the supremacy of this proposed method over the other conventional methods.

The change in reactance of all lines after an outage in line 1 - 2 is tabulated in Table 4 and the line reactance gets changed to a value to accommodate the change in system state resulting from a contingency. Then, the same procedure as mentioned above is followed and the cost deviations due to generation rescheduling patterns for various contingencies i.e. outage of lines 1 - 2, 1 - 3 and 19 - 20 are obtained one by one and the results are summarized in Table 5.

It is proved that there is a notable improvement in cost due to the use of FTLI as a corrective variable. Particularly, the post contingency cost deviation is negative in CASE 3B and it indicates a reduction in cost even after an outage. It is also worth to mention that the percentage improvement is more than 100 in such case, which indicates the effectiveness of flexibility offered by the transmission system.

\section{Conclusion}

In this work, the incorporation of FTLI into CSCOPF was proposed, in which the line reactance is taken as a control variable and it is varied optimally with respect to contingencies. The proposed idea is tested in IEEE 30 bus system and the results have proved that improvement in cost and reduction in rescheduling are phenomenal. The idea, which is used to view the transmission system as a dynamic one rather than a static one, helps the transmission system to contribute in ensuring security, stability and reliability of the overall system. It is suggested that, the proper incorporation of FTLI in power system optimization is certainly a better solution for meeting the increase in power demand without the extension of transmission system. Next, the work is to be extended by deriving the sensitivities (gradient) of the system equations with respect to line reactance, so that a correlation can be established between the operation of the power system and the line reactance. These sensitivities are to be used for application in the process of optimizing the system performance.

\section{Acknowledgements}

The authors of this manuscript express their sincere thanks to the Management of Kamaraj College of Engineer- 
Table 3. Generation and cost output for case 1B.

\begin{tabular}{|c|c|c|c|c|c|}
\hline \multirow{2}{*}{$\begin{array}{c}\text { Gen. Bus } \\
\text { No. }\end{array}$} & \multicolumn{2}{|c|}{ Before the outage } & \multicolumn{2}{|c|}{ After the outage } & \multirow{2}{*}{ Cost deviation $(\$ / \mathrm{hr})$} \\
\hline & Generation (MW) & Cost $(\$ / h r)$ & Generation (MW) & Cost $(\$ / h r)$ & \\
\hline 1 & 199.28 & \multirow{6}{*}{8915.04} & 130.00 & \multirow{6}{*}{9448.23} & \multirow{6}{*}{533.20} \\
\hline 2 & 37.22 & & 40.82 & & \\
\hline 5 & 35.99 & & 64.33 & & \\
\hline 8 & 15.09 & & 29.29 & & \\
\hline 11 & 6.33 & & 27.12 & & \\
\hline 13 & 0 & & 1.95 & & \\
\hline
\end{tabular}

Table 4. The change in reactance of various lines after outage.

\begin{tabular}{|c|c|c|c|c|c|c|c|}
\hline Branch No. & $\begin{array}{l}\text { Reactance before } \\
\text { outage } \\
\text { (p.u.) }\end{array}$ & $\begin{array}{l}\text { Reactance after } \\
\text { outage } \\
\text { (p.u.) }\end{array}$ & $\begin{array}{l}\text { Change in } \\
\text { reactance in \% }\end{array}$ & Branch No. & $\begin{array}{l}\text { Reactance before } \\
\text { outage (p.u.) }\end{array}$ & $\begin{array}{l}\text { Reactance after } \\
\text { outage } \\
\text { (p.u.) }\end{array}$ & $\begin{array}{l}\text { Change in } \\
\text { reactance in \% }\end{array}$ \\
\hline 1 & 0.0575 & 0.0575 & 0.00 & 22 & 0.2185 & 0.1819 & -16.76 \\
\hline 2 & 0.1652 & 0.1610 & -2.55 & 23 & 0.1292 & 0.1550 & 20.00 \\
\hline 3 & 0.1737 & 0.2084 & 20.00 & 24 & 0.0680 & 0.0613 & -9.90 \\
\hline 4 & 0.0379 & 0.0455 & 20.00 & 25 & 0.2090 & 0.1672 & -20.00 \\
\hline 5 & 0.1983 & 0.1586 & -20.00 & 26 & 0.0845 & 0.1014 & 20.00 \\
\hline 6 & 0.1763 & 0.2116 & 20.00 & 27 & 0.0749 & 0.0599 & -20.00 \\
\hline 7 & 0.0414 & 0.0478 & 15.58 & 28 & 0.1499 & 0.1215 & -18.96 \\
\hline 8 & 0.1160 & 0.1392 & 20.00 & 29 & 0.0236 & 0.0283 & 20.00 \\
\hline 9 & 0.0820 & 0.0830 & 1.22 & 30 & 0.2020 & 0.1616 & -20.00 \\
\hline 10 & 0.0420 & 0.0350 & -16.74 & 31 & 0.1790 & 0.2015 & 12.59 \\
\hline 11 & 0.2080 & 0.2496 & 20.00 & 32 & 0.2700 & 0.3240 & 20.00 \\
\hline 12 & 0.5560 & 0.6672 & 20.00 & 33 & 0.3292 & 0.3950 & 20.00 \\
\hline 13 & 0.2080 & 0.1664 & -20.00 & 34 & 0.3800 & 0.3040 & -20.00 \\
\hline 14 & 0.1100 & 0.1320 & 20.00 & 35 & 0.2087 & 0.2504 & 20.00 \\
\hline 15 & 0.2560 & 0.2048 & -20.00 & 36 & 0.3960 & 0.3168 & -20.00 \\
\hline 16 & 0.1400 & 0.1120 & -20.00 & 37 & 0.4153 & 0.3322 & -20.00 \\
\hline 17 & 0.2559 & 0.2047 & -20.00 & 38 & 0.6027 & 0.4822 & -20.00 \\
\hline 18 & 0.1304 & 0.1043 & -20.00 & 39 & 0.4533 & 0.3626 & -20.00 \\
\hline 19 & 0.1987 & 0.1590 & -20.00 & 40 & 0.2000 & 0.1790 & -10.48 \\
\hline 20 & 0.1997 & 0.2396 & 20.00 & 41 & 0.0599 & 0.0479 & -20.00 \\
\hline 21 & 0.1923 & 0.1538 & -20.00 & - & - & - & - \\
\hline
\end{tabular}

Table 5. Comparison of results with various contingencies.

\begin{tabular}{|c|c|c|c|c|c|}
\hline Case & $\begin{array}{c}\text { Line that is put out } \\
\text { of service }\end{array}$ & $\begin{array}{l}\text { Cost deviation without } \\
\text { FTLI (A) \$/hr }\end{array}$ & $\begin{array}{l}\text { Cost deviation with } \\
\text { FTLI } \\
\text { (B) } \$ / h r\end{array}$ & $\begin{array}{c}\text { Improvement in cost } \\
\text { due to FTLI } \\
\$ / \mathrm{hr}\end{array}$ & $\begin{array}{c}\text { Percentage } \\
\text { improvement in } \\
\text { cost } \%\end{array}$ \\
\hline 1 & 1 & 575.34 & 533.20 & 42.14 & 7.3 \\
\hline 2 & 5 & 169.23 & 111.62 & 57.14 & 34 \\
\hline 3 & 15 & 37.26 & -5.25 & 42.50 & 114 \\
\hline
\end{tabular}


ing \& Technology and the authorities of Anna University Regional Campus Madurai for their support to complete this research.

\section{References}

[1] Alsac, O. and Stott, B. (1974) Optimal Load Flow with Steady State Security. IEEE Transactions on Power Apparatus and Systems, PAS-93, 745-751. http://dx.doi.org/10.1109/TPAS.1974.293972

[2] Monticelli, A., Pereira, M.V.F. and Granville, S. (1987) Security Constrained Optimal Power Flow with Post Contingency Rescheduling. IEEE Transactions on Power Systems, PWRS-2, 175-180. http://dx.doi.org/10.1109/TPWRS.1987.4335095

[3] Momoh, J.A., Zhu, J.Z., Boswell G.D. and Hoffman, S. (2001) Power System Security Enhancement by OPF with Phase Shifter. IEEE Transactions on Power Systems, 16, 287-293. http://dx.doi.org/10.1109/59.918300

[4] Thomas, J.J. and Grijalva, S. (2014) Flexible Security Constrained Optimal Power Flow. IEEE Transactions on Power Systems, 30, 1195-1202. http://dx.doi.org/10.1109/TPWRS.2014.2345753

[5] Hedman, K.W., O’Neill, R.P., Fisher, E.B. and Oren, S.S. (2009) Optimal Transmission Switching with Contingency Analysis. IEEE Transactions on Power Systems, 24, 1577-1586. http://dx.doi.org/10.1109/TPWRS.2009.2020530

[6] Phan, D.T. and Sun, X.A. (2014) Minimal Impact Corrective Actions in Security Constrained Optimal Power Flow via Sparsity Regularization. IEEE Transactions on Power Systems, 30, 1947-1956. http://dx.doi.org/10.1109/TPWRS.2014.2357713

[7] Zhang, R., Dong, Z.Y., Xu, Y., Wong, K.P. and Lai, M.M. (2014) Hybrid Computation of Corrective Security Constrained Optimal Power Flow Problems. IET Generation Transmission Distribution, 8, 995-1006. http://dx.doi.org/10.1049/iet-gtd.2013.0384

[8] Dash, P.K., Pradhan, A.K., Panda, G. and Liew, A.C. (2000) Adaptive Relay Setting for Flexible AC Transmission Systems (FACTS). IEEE Transactions on Power Delivery, 15, 38-43. http://dx.doi.org/10.1109/61.847226

[9] Chung, T.S. and Li, Y.Z. (2000) A Hybrid GA Approach for OPF with Consideration of FACTS Devices. IEEE Power Engineering Review, 20, 54-57. http://dx.doi.org/10.1109/39.857456

[10] Sahraei-Ardakani, M. and Hedman, K.W. (2014) A Fast LP Approach for Enhanced Utilization of Variable Impedance Based FACTS Devices. IEEE Transactions on Power Systems, 66, 1-10.

[11] Ding, T., Bo, R., Li, F. and Sun, H. (2015) Optimal Power Flow with the Consideration of Flexible Transmission Line Impedance. IEEE Transactions on Power Systems, 31, 1655-1656. http://dx.doi.org/10.1109/TPWRS.2015.2412682

[12] Zimmerman, R.D., Murillo-Sánchez, C.E. and Thomas, R.J. (2011) MATPOWER: Steady-State Operations, Planning, and Analysis Tools for Power Systems Research and Education. IEEE Transactions on Power Systems, 26, 12-19. http://dx.doi.org/10.1109/TPWRS.2010.2051168 